\title{
Tumor necrosis factor- $\alpha$ promotes the expression of excitatory amino-acid transporter 2 in astrocytes: Optimal concentration and incubation time
}

\author{
YUEMIN DING ${ }^{1,2^{*}}$, KENA ZHANG ${ }^{3 *}$, SHUQIN LIU $^{4}$, QIJUN ZHANG ${ }^{2}$, \\ CHIYUAN MA ${ }^{2}$, IAIN C. BRUCE ${ }^{2}$ and XIONG ZHANG ${ }^{2}$ \\ ${ }^{1}$ School of Medicine, Zhejiang University City College, Hangzhou, Zhejiang 310015; \\ ${ }^{2}$ Department of Basic Medicine, College of Medicine, Zhejiang University, Hangzhou, Zhejiang 310058; \\ ${ }^{3}$ College of Basic Medical Science, Zhejiang Chinese Medical University, Hangzhou, Zhejiang 310053; \\ ${ }^{4}$ Department of Pharmacy, Wannan Medical College, Wuhu, Anhui 241002, P.R. China
}

Received April 4, 2014; Accepted September 8, 2014

DOI: $10.3892 /$ etm.2014.2024

\begin{abstract}
The aim of the present study was to determine whether tumor necrosis factor (TNF)- $\alpha$ regulates the expression levels of excitatory amino-acid transporters (EAATs) in primary astrocytes and its roles in brain ischemia. Exogenous TNF- $\alpha$ was administered to pure cultured astrocytes and the expression levels of EAAT1, EAAT2 and glial fibrillary acidic protein (GFAP) were evaluated. The results showed that TNF- $\alpha$ at $10 \mathrm{ng} / \mathrm{ml}$ enhanced the expression of EAAT2 in a time-dependent manner, while the expression levels of EAAT1 and GFAP did not change. To determine whether the elevation in the levels of the EAAT2 protein induced by TNF- $\alpha$ had a beneficial effect on ischemic insult, TNF- $\alpha$ was applied to in vitro models of cerebral ischemia; the treatment was observed to increase neuronal viability. The present results suggest that a relatively short-term application of an optimal concentration of TNF- $\alpha$ may protect neurons against ischemic injury by elevating the expression of EAAT2 in astrocytes.
\end{abstract}

\section{Introduction}

Acute ischemic stroke is one of the leading causes of adult disability (1). The excitatory neuronal toxicity caused by the accelerated release of glutamate during brain ischemia is the key element leading to neuronal death. The concentration of glutamate in the synaptic cleft is regulated by membrane-bound

Correspondence to: Dr Xiong Zhang, Department of Basic Medicine, College of Medicine, Zhejiang University, Research Building C514, 866 Yu Hang Tang Road, Hangzhou, Zhejiang 310058, P.R. China

E-mail: xiongzhang@zju.edu.cn

*Contributed equally

Key words: tumor necrosis factor- $\alpha$, excitatory amino-acid transporter 2, astrocyte, ischemia excitatory amino-acid transporters (EAATs) (1). To date, five subtypes have been identified and termed EAAT1-5 in human tissues. Glutamate/aspartate transporter, glutamate transporter-1 and excitatory amino-acid carrier 1 are the rodent homologs of EAAT1, 2 and 3, respectively (3). EAAT2 is primarily an astrocytic transporter and is highly expressed throughout the central nervous system (CNS), removing $>90 \%$ of the total extracellular glutamate from the synaptic cleft (4).

Tumor necrosis factor- $\alpha$ (TNF- $\alpha$ ) is an important inflammatory factor and its expression levels markedly increase in the brain following ischemia (5). For a long period of time, TNF- $\alpha$ was believed to induce neuronal necrosis, resulting in brain injury (6), until Cheng et al (7) demonstrated that TNF- $\alpha$ (1-100 $\mathrm{ng} / \mathrm{ml})$ pretreatment protected cultured neurons from glucose deprivation-induced injury and excitatory amino-acid toxicity. By knocking down the receptors for TNF- $\alpha$ in mice, Gary et al (8) unexpectedly observed more severe neuronal damage caused by focal brain ischemia, leading to the hypothesis that TNF- $\alpha$ exhibits a protective role on ischemic neurons. Since then, increasing evidence has shown that TNF- $\alpha$ plays a dual role in ischemic neuronal injury by inducing necrosis and protecting injured neurons (9-12). Our previous study demonstrated that the permeability of the blood-brain barrier to TNF- $\alpha$ transiently increased following injury to the CNS (13). Notably, the time-course of the TNF- $\alpha$ permeability was in accordance with that of the functional recovery, which indicated that the optimal concentration and timing of TNF- $\alpha$ administration contribute to neuroprotection. Although different mechanisms have been proposed to explain the dual role of TNF- $\alpha$ (10-12), the association between TNF- $\alpha$ and EAATs on astrocytes remains unclear. The aim of the present study was to determine whether TNF- $\alpha$ regulates the expression level of EAAT2 in primary astrocytes in culture and its role in brain ischemia.

\section{Materials and methods}

Animals and reagents. Adult male Sprague Dawley rats were obtained from the Experimental Animal Centre of Zhejiang 
University (Hangzhou, China). The experimental procedures were approved by the Animal Ethics Committee of Zhejiang University and were carried out in accordance with institutional guidelines. Recombinant rat TNF- $\alpha$ was obtained from BioVision (Milpitas, CA, USA).

Primary hippocampal neuron culture. Rat hippocampal neurons were prepared as previously described, with certain modifications (14). Briefly, the hippocampi of E18 embryos were dissected and dissociated in oxygenated Hank's balanced salt solution. Cells $\left(1 \times 10^{6}\right)$ were plated on coverslips coated with poly-D-lysine $(50 \mu \mathrm{g} / \mathrm{ml}$; Sigma-Aldrich, St. Louis, MO, USA) or on coverslips in Neurobasal ${ }^{\circledR}$ medium supplemented with 2\% B27 (Invitrogen Life Technologies, San Diego, CA, USA) and $0.2 \mathrm{mM} \mathrm{L-glutamine} \mathrm{(Sigma-Aldrich.}$ Cytosine-D-arabinoside (10 $\mu \mathrm{M}$; Sigma-Aldrich) was added to the cultures two days after plating to block the proliferation of non-neuronal cells. One-half of the culture medium was changed every four days. After culturing for 12 days, neurons were then co-cultured with astrocytes.

Primary cortical astrocyte culture and TNF- $\alpha$ treatment. Astrocytes in primary culture were prepared from the cerebral cortices of newborn (0-12 h postnatal) rat pups as previously described (15). In brief, cells were trypsinized with $0.25 \%$ trypsin (Invitrogen Life Technologies, Carlsbad, CA, USA) for $16 \mathrm{~min}$, followed by trituration with $10 \mathrm{mg} / 1 \mathrm{DNAse}$ (Sigma-Aldrich). Cells were plated in poly-D-lysine-coated flasks and maintained in Dulbecco's Modified Eagle's medium (DMEM; Invitrogen Life Technologies) supplemented with $10 \%$ fetal bovine serum (PAA, Pasching, Austria). The culture medium was changed every third day. After nine days, the flasks were agitated for $12 \mathrm{~h}$ at $37^{\circ} \mathrm{C}$ to separate the microglia from the more adherent mass of astrocytes. The adherent cells were replated in dishes and cultured for another week prior to experimentation. Immunostaining with the glial fibrillary acidic protein (GFAP) antibody (Cell Signaling Technology, Inc., Danvers, MA, USA) confirmed that the majority (>95\%) of the cells expressed GFAP (data not shown). To examine the dose-dependent effect, recombinant rat TNF- $\alpha(1,10,20$ and $50 \mathrm{ng} / \mathrm{ml}$ ) was added directly to the medium and the cells were lysed following 24-h incubation. To explore the timedependent effect, astrocytes were treated with $10 \mathrm{ng} / \mathrm{ml} \mathrm{TNFa}$ for $4,8,12,24,36$ or $72 \mathrm{~h}$ and lysed after treatment.

Co-culture of astrocytes and neurons. Co-cultures of astrocytes and neurons were prepared as previously described, with certain modifications (16). Briefly, astrocytes in Petri dishes were washed twice with phosphate-buffered saline (PBS) and incubated in serum-free medium for $3 \mathrm{~h}$. Glass coverslips with hippocampal neurons were inverted over the separately-cultured astrocytes so that the neurons were facing the astrocytes. The cells were incubated together in Neurobasal medium containing B27 and L-glutamine for $48 \mathrm{~h}$ to induce direct contact. The cultures were pretreated with $10 \mathrm{ng} / \mathrm{ml} \mathrm{TNF}-\alpha$ for $0-24 \mathrm{~h}$ prior to being subjected to oxygen-glucose deprivation (OGD).

$O D G$. OGD was performed as previously described (17). Briefly, co-cultured cells were washed with PBS and incubated in glucose-free DMEM. The cultures were transferred to an anaerobic chamber filled with a gas mixture of $95 \% \mathrm{~N}_{2}$ and $5 \% \mathrm{CO}_{2}$ at $37^{\circ} \mathrm{C}$ for $2 \mathrm{~h}$. Exposure was terminated by removing the glucose-free DMEM and adding Neurobasal medium. After $22 \mathrm{~h}$ of recovery in a normoxic incubator, the coverslips were carefully transferred, face-up, to a new 24-well plate containing Neurobasal medium and the cell viability was assessed as described below. In each experiment, cultures exposed to OGD were compared with normoxic controls supplied with DMEM containing glucose and maintained in standard incubation conditions.

MTT assay. Cell viability was measured using the MTT (Sigma-Aldrich) assay. Cells were gently washed with fresh culture medium, and DMEM and the serum-free medium containing MTT $(5 \mathrm{mg} / \mathrm{ml})$ was added and incubated for an additional $4 \mathrm{~h}$ at $37^{\circ} \mathrm{C}$. The purple-blue MTT formazan precipitate was dissolved in $150 \mu \mathrm{l}$ dimethyl sulfoxide. Absorbance was measured at $490 \mathrm{~nm}$.

$T N F-\alpha$ treatment in vivo. A total of 6 male rats were anesthetized by an intraperitoneal injection of $2 \%$ sodium pentobarbital $(40 \mathrm{mg} / \mathrm{kg})$ and mounted in a stereotaxic frame on a heating blanket. TNF- $\alpha$ was administered according to previously described techniques (18), with certain modifications. Intracerebroventricular (i.c.v.) injection was performed at the following coordinates: $0.6 \mathrm{~mm}$ posterior, $4.5 \mathrm{~mm}$ ventral and $1.6 \mathrm{~mm}$ lateral to the bregma. Recombinant rat TNF- $\alpha$ $(100 \mathrm{ng} / 15 \mu \mathrm{l})$ was injected into the left lateral ventricle over a period of $10 \mathrm{~min}$. A total of 3 rats in the control group received the same volume of sterilized, artificial cerebrospinal fluid. The expression levels of the EAATs in the cerebral cortex were determined by western blot analysis $24 \mathrm{~h}$ after treatment with TNF- $\alpha$.

Western blot assay. Cerebral cortical tissues or astrocytes were lysed on ice in radioimmunoprecipitation assay buffer with a protease inhibitor cocktail (Sigma-Aldrich) containing a mixture of several protease inhibitors, including AEBSF at $104 \mathrm{mM}$, Aprotinin at $80 \mu \mathrm{M}$, Bestatin at $4 \mathrm{mM}, \mathrm{E}-64$ at $1.4 \mathrm{mM}$, Leupeptin at $2 \mathrm{mM}$ and Pepstatin A at $1.5 \mathrm{mM}$. Protein content was estimated using the bicinchoninic acid assay method. Immunoblotting was performed as described previously (19). Following electrophoresis, protein samples were transferred to polyvinylidene fluoride membranes. The membranes were incubated at $4^{\circ} \mathrm{C}$ overnight with antibodies against EAAT1 (1:500; Wuhan Boster Biological Technology, Ltd., Wuhan, China), EAAT2 (1:500; Wuhan Boster Biological Technology, Ltd.), GFAP $(1: 2,000)$ or $\beta$-actin $(1: 1,000$; Cell Signaling Technology, Inc.) diluted in blocking solution. Following $2 \mathrm{~h}$ of incubation with a horseradish peroxidase-labeled secondary antibody (Jackson ImmunoResearch Laboratories, West Grove, PA, USA) at room temperature, the blots were developed with enhanced chemiluminescence reagents (Pierce, Rockford, IL, USA) and exposed to X-ray film to obtain images. The intensity of each band was quantified using ImageJ software (National Institutes of Health, Bethesda, MD, USA) and normalized to $\beta$-actin.

Statistical analysis. Data are expressed as the mean \pm standard deviation. Comparisons between groups were analyzed using 
A

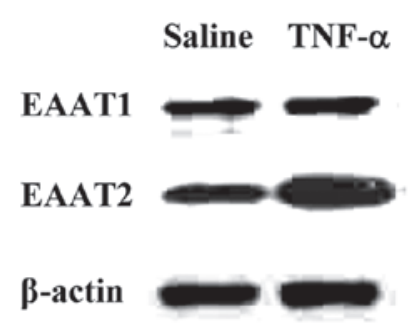

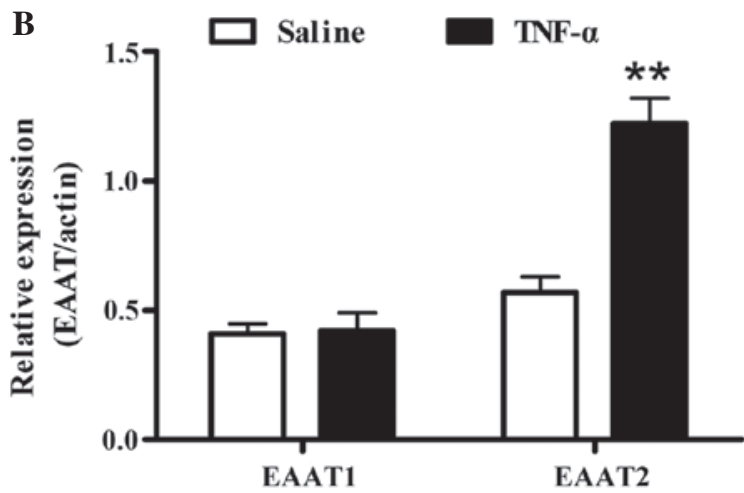

Figure 1. TNF- $\alpha$ treatment elevates the expression of EAAT2 in the brain. (A) Expression levels of the EAATs following TNF- $\alpha$ treatment. (B) Quantification of the expression levels of the EAATs. Data are presented as the mean \pm standard deviation and were analyzed by one-way analysis of variance $(\mathrm{n}=3)$. ${ }^{* *} \mathrm{P}<0.01$ compared with saline controls. TNF- $\alpha$, tumor necrosis factor- $\alpha$; EAAT, excitatory amino-acid transporter.

the Student's t-test or one-way analysis of variance, followed by Dunnett's post hoc test. $\mathrm{P}<0.05$ was considered to indicate a statistically significant difference.

\section{Results}

TNF- $\alpha$ elevates EAAT2 expression in vivo. To investigate the effect of TNF- $\alpha$ on the expression of EAATs in vivo, recombinant rat TNF- $\alpha$ was administered by i.c.v. injection and the expression levels of the EAATs in the cerebral cortical tissue were assessed by western blot analysis. Compared with the EAAT2 levels in the saline control group, EAAT2 expression markedly increased $(\mathrm{P}<0.01)$ after TNF- $\alpha$ treatment for $24 \mathrm{~h}$. However, no changes were observed in the expression level of EAAT1 (Fig. 1).

TNF- $\alpha$ regulates EAAT2 expression in astrocytes. To determine whether the expression levels of EAAT proteins in astrocytes are regulated by $\mathrm{TNF}-\alpha$, the dose-dependent effect of TNF- $\alpha(1,10,20 \mathrm{or} 50 \mathrm{ng} / \mathrm{ml})$ on primary cortical astrocytes was analyzed (Fig. 2). The optimal concentration of TNF- $\alpha$ (10 ng/ml) upregulated EAAT2 expression $(\mathrm{P}<0.01)$, whereas a high concentration of TNF- $\alpha(50 \mathrm{ng} / \mathrm{ml})$ negatively regulated EAAT2 expression $(\mathrm{P}<0.05)$. Different doses of TNF- $\alpha$ did not change the expression levels of EAAT1 or GFAP, the latter representing astrocyte proliferation (Fig. 2).

To further explore the association between incubation time and the expression levels of EAATs in astrocytes, the astrocytes were treated with the optimal concentration of TNF- $\alpha$ $(10 \mathrm{ng} / \mathrm{ml})$ for different incubation periods $(4,8,12,24,36$ or $72 \mathrm{~h})$. The EAAT2 protein expression showed a time-dependent increase, followed by a time-dependent decrease, with a maximum level $(\mathrm{P}<0.01)$ after $12 \mathrm{~h}($ Fig. 3). However, the expression levels of EAAT1 and GFAP did not change with treatment. These results indicated that short-term treatment with the optimal concentration of TNF- $\alpha$ promoted EAAT2 expression in astrocytes, which was independent of astrocyte proliferation.

TNF- $\alpha$ treatment increases neuronal viability following $O G D$ injury. To determine whether TNF- $\alpha$ treatment is neuroprotective against neuronal ischemic injury in vitro, a co-culture system of astrocytes and neurons was established and cell
A TNF- $\alpha(\mathrm{ng} / \mathrm{ml})$

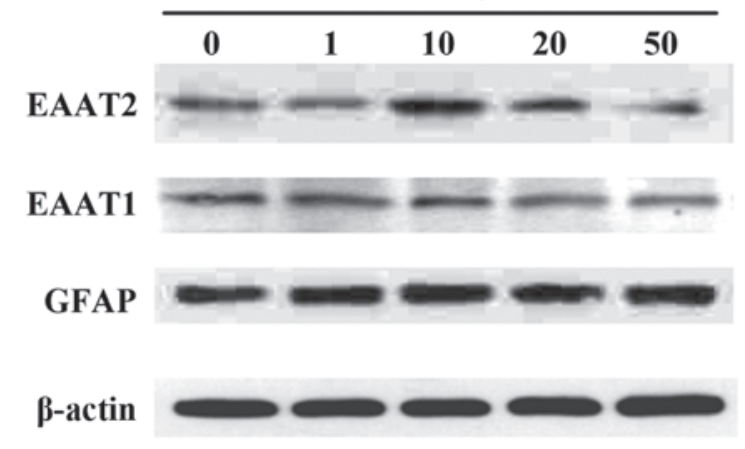

B

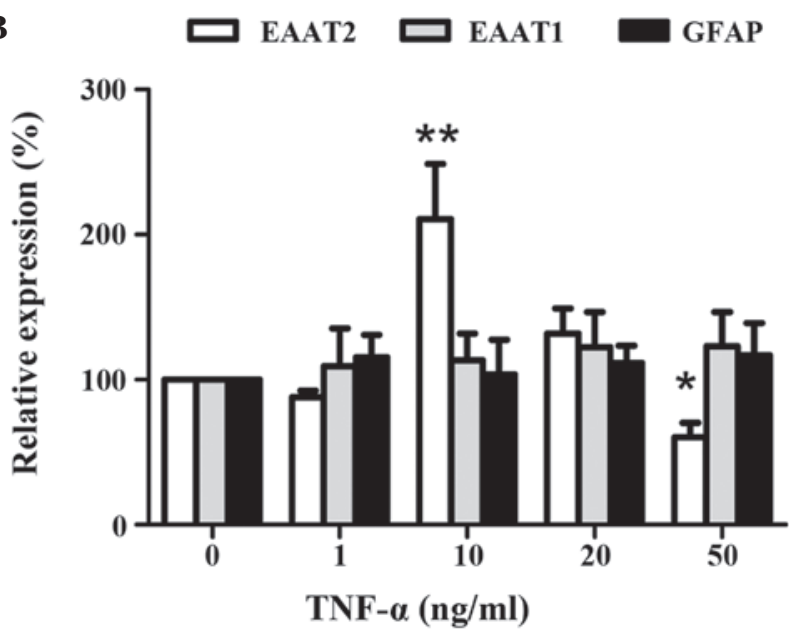

Figure 2. Expression of EAATs in astrocytes treated with different doses of TNF- $\alpha$. (A) Representative western blot analysis of EAAT1, EAAT2, GFAP and $\beta$-actin. (B) Densitometric analysis showing the expression of EAATs or GFAP normalized to $\beta$-actin. Data are presented as the mean \pm standard deviation $(\mathrm{n}=5) .{ }^{*} \mathrm{P}<0.05$ and ${ }^{* *} \mathrm{P}<0.01$ compared with the control group $(0 \mathrm{ng} / \mathrm{ml}$ TNF- $\alpha$ ). TNF- $\alpha$, tumor necrosis factor- $\alpha$; EAAT, excitatory amino-acid transporter; GFAP, glial fibrillary acidic protein.

viability was assessed following OGD injury. After different periods of incubation ( $0-24 \mathrm{~h}$ ) with $10 \mathrm{ng} / \mathrm{ml} \mathrm{TNF}-\alpha$, OGD was applied for $2 \mathrm{~h}$, followed by $22 \mathrm{~h}$ of reoxygenation (Fig. 4A). An MTT assay was performed on the hippocampal neurons to reveal the cell viability following OGD insult. The results 
A
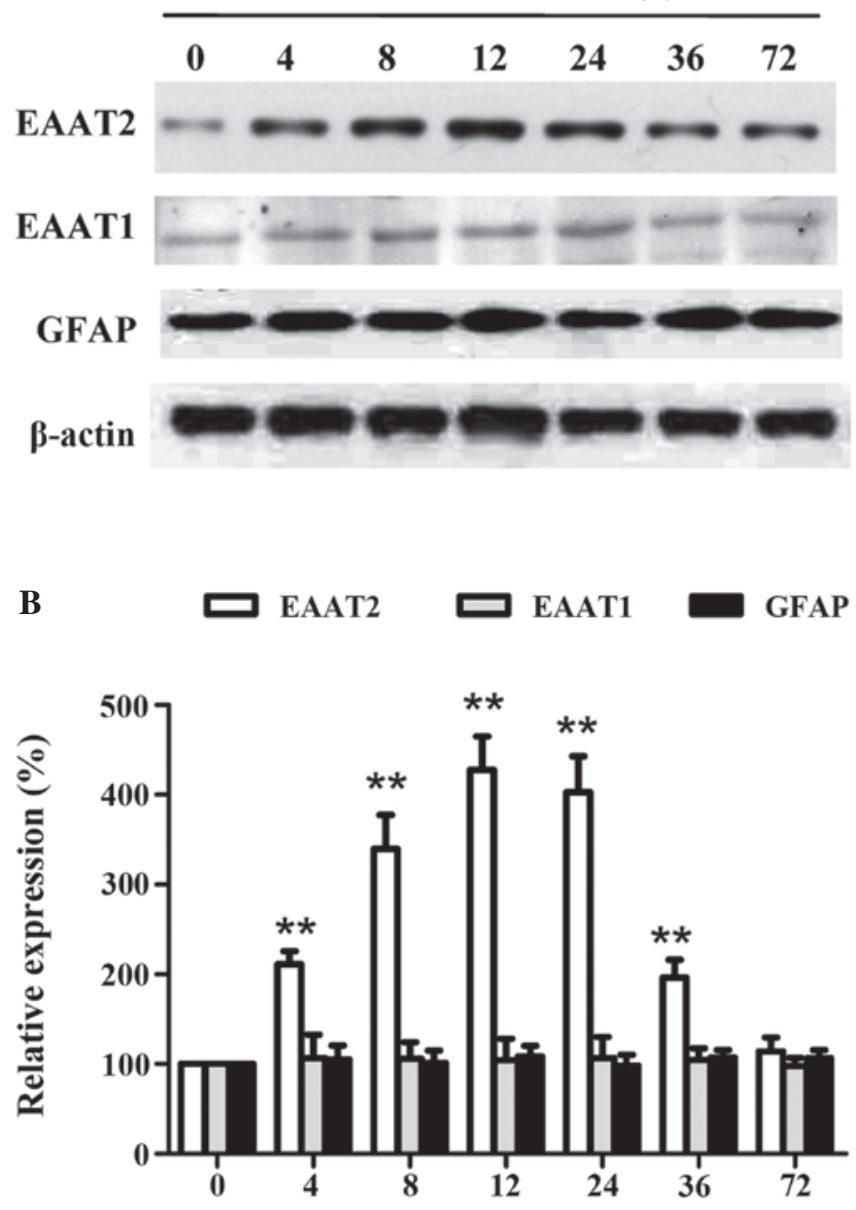

TNF- $\alpha$ incubation time (h)

Figure 3. Expression levels of EAATs in astrocytes treated with TNF- $\alpha$ for different time-periods. (A) Representative western blot analysis of EAAT1, EAAT2, GFAP and $\beta$-actin. (B) Densitometric analysis showing the expression levels of the EAATs or GFAP normalized to $\beta$-actin. Data are presented as the mean \pm standard deviation $(\mathrm{n}=6)$. ${ }^{* *} \mathrm{P}<0.01$ compared with the control group (prior to TNF- $\alpha$ treatment, $0 \mathrm{~h}$ ). TNF- $\alpha$, tumor necrosis factor- $\alpha$ EAAT, excitatory amino-acid transporter; GFAP, glial fibrillary acidic protein.

demonstrated that only short-term (4 h) TNF- $\alpha$ treatment led to a significantly higher cell viability value compared with the other groups $(\mathrm{P}<0.01)$ (Fig. 4B).

\section{Discussion}

Clinical studies have demonstrated that a transient ischemic attack (TIA) within a narrow time-window may enhance the tolerance of the brain against a more severe ischemic insult (20-22). Notably, elevation in the plasma levels of TNF- $\alpha$ have been reported in patients for $<72 \mathrm{~h}$ following a TIA (23), leading to the proposal that TNF- $\alpha$ pretreatment may mimic the neuroprotective effect of ischemic preconditioning. Coinciding with this hypothesis, it has been found that the elevation in TNF- $\alpha$ levels induced by ischemic preconditioning and direct TNF- $\alpha$ pretreatment contributes to the enhancement of cellular defense against the second insult $(10,15,24)$. In the present study, in vivo TNF- $\alpha$ treat-
A
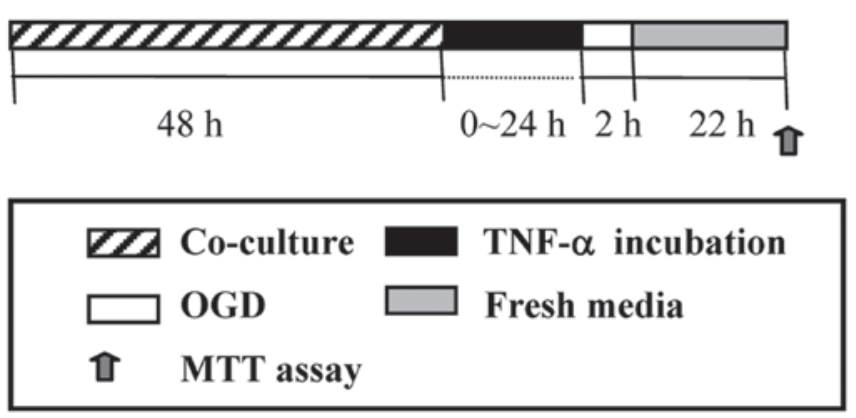

B

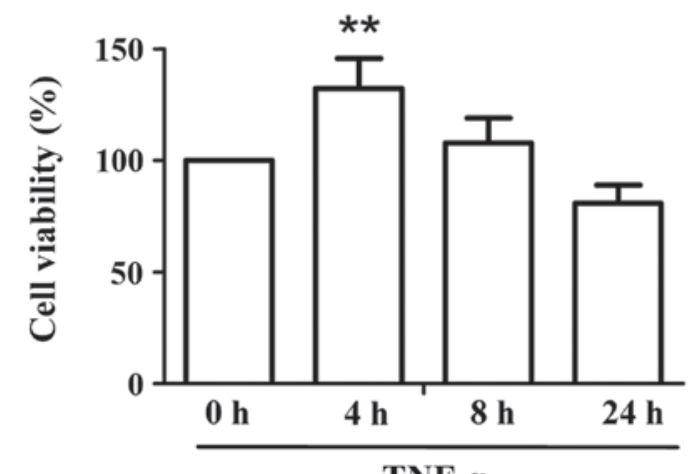

Figure 4. Effect of TNF- $\alpha$ pretreatment on neuronal viability following OGD. (A) Timeline of the experimental procedure. At nine days in vitro the neuron and astrocyte co-cultures were set up and maintained for $48 \mathrm{~h}$. The cultures were pretreated with $10 \mathrm{ng} / \mathrm{ml} \mathrm{TNF}-\alpha$ for $0-24 \mathrm{~h}$ prior to OGD and subsequently placed in fresh medium for $22 \mathrm{~h}$ to recover. Neuronal viability was measured by MTT assay. (B) MTT results showing that short-term (4 h) TNF- $\alpha$ pretreatment had a distinct neuroprotective effect against OGD injury. Data are presented as the mean \pm standard deviation $(n=5) .{ }^{* *} \mathrm{P}<0.01$ compared with the corresponding 0 h group. TNF- $\alpha$, tumor necrosis factor- $\alpha$; OGD, oxygen-glucose deprivation.

ment markedly decreased the infarct volume in the ischemic brain and improved the functional recovery of rats receiving a subsequent middle cerebral artery occlusion (data not shown).

Numerous studies have been performed to investigate the mechanisms of ischemic tolerance caused by TNF- $\alpha$ exposure. Glazner and Mattson found that TNF- $\alpha$ pretreatment reduced calcium influx and upregulated the gene transcription of neuroprotective factors (25). In addition, Watters and O'Conner (26) and Watters et al (27) revealed that TNF- $\alpha$ pretreatment increased the resistance of neurons to a subsequent insult from glutamate by attenuating resting calcium activity and calcium-related responsiveness. In the present study, the expression level of EAAT2, but not EAAT1, was upregulated by TNF- $\alpha$ treatment in vivo and in vitro. This was consistent with the findings of Davis and Patel (28), who demonstrated that the expression level of EAAT2 was significantly increased in ischemic preconditioning, suggesting that EAAT2 upregulation attenuates the toxicity of the excitatory glutamate induced by a more severe ischemic insult. This was further confirmed by the study of Weller et al (29), in which it was observed that astrocytes that had undergone transfection to overexpress EAAT2 played a marked protective role against moderate ischemia.

Consistent with the present results, Tilleux et al (30) reported the stimulatory effects of TNF- $\alpha$ on EAAT2 expres- 
sion, although other studies have found inhibitory effects. $\mathrm{Su}$ et al (31) revealed that the mRNA expression of EAAT2 began to decline from $48 \mathrm{~h}$ with TNF- $\alpha(30 \mathrm{ng} / \mathrm{ml})$ treatment. Thus, the effect of TNF- $\alpha$ on EAAT2 expression remains controversial. In the present study, TNF- $\alpha$ treatment promoted the expression of EAAT2 in astrocytes in a concentrationand time-dependent manner. The current results suggested that a lower concentration $(<20 \mathrm{ng} / \mathrm{ml})$ of TNF- $\alpha$ applied as a short-term $(<36 \mathrm{~h})$ treatment contributed to the induction of a neuroprotective effect by elevating EAAT2 expression, while this effect was lost or even reversed with increasing concentration and incubation time. Although the underlying mechanism remains unclear, this may explain why certain studies provide evidence for an inhibitory influence of TNF- $\alpha$ on EAAT2 expression. Furthermore, it was demonstrated in the present study that incubation with TNF- $\alpha$ did not increase astrocyte proliferation. Therefore, the elevated expression of EAAT2 in astrocytes was proliferation-independent.

To further determine whether the elevated levels of EAAT2 protein induced by TNF- $\alpha$ had a beneficial outcome in vitro, the viability following OGD injury in a co-culture system of astrocytes and neurons was assessed. Corresponding with the in vivo results, it was revealed that TNF- $\alpha$ increased the cell viability following OGD injury, suggesting that it has a neuroprotective effect by promoting EAAT2 expression in astrocytes.

The present study had several limitations. Firstly, based on previous findings, TNF- $\alpha$ may have dual effects on the expression and activity of EAATs. Thus, detection of the activity of EAATs under these experimental conditions is required. Secondly, it is necessary to demonstrate whether the protection is eradicated following the blockade of glutamate uptake. Finally, an advanced mechanistic study is also required. Therefore, these issues should be addressed in future studies.

In conclusion, the present study revealed that an optimal concentration and time-course of TNF- $\alpha$ elevates EAAT2 expression in astrocytes and has a beneficial effect on subsequent ischemic insult.

\section{Acknowledgements}

This study was supported by the Natural Science Foundation of Zhejiang Province (Y2100508) and the National Natural Science Foundation of China (81000535).

\section{References}

1. Krishnamurthi RV, Feigin VL, Forouzanfar MH, et al; GBD Stroke Experts Group. Global and regional burden of first-ever ischaemic and haemorrhagic stroke during 1990-2010: findings from the Global Burden of Disease Study 2010. Lancet Glob Health 1: e259-e281, 2013.

2. Chao XD, Fei F and Fei Z: The role of excitatory amino acid transporters in cerebral ischemia. Neurochem Res 35: 1224-1230,2010

3. Bunch L, Erichsen MN and Jensen AA: Excitatory amino acid transporters as potential drug targets. Expert Opin Ther Targets 13: 719-731, 2009.

4. Maragakis NJ and Rothstein JD: Glutamate transporters: animal models to neurologic disease. Neurobiol Dis 15: 461-473, 2004.

5. Botchkina GI, Meistrell ME III, Botchkina IL and Tracey KJ: Expression of TNF and TNF receptors (p55 and p75) in the rat brain after focal cerebral ischemia. Mol Med 3: 765-781, 1997.

6. Feuerstein G, Wang X and Barone FC: Cytokines in brain ischemia - the role of TNF alpha. Cell Mol Neurobiol 18: 695-701, 1998.
7. Cheng B, Christakos S and Mattson MP: Tumor necrosis factors protect neurons against metabolic-excitotoxic insults and promote maintenance of calcium homeostasis. Neuron 12: 139-153, 1994.

8. Gary DS, Bruce-Keller AJ, Kindy MS and Mattson MP: Ischemic and excitotoxic brain injury is enhanced in mice lacking the p55 tumor necrosis factor receptor. J Cereb Blood Flow Metab 18: 1283-1287, 1998.

9. Lambertsen KL, Clausen BH, Babcock AA, et al: Microglia protect neurons against ischemia by synthesis of tumor necrosis factor. J Neurosci 29: 1319-1330, 2009.

10. Saha RN, Liu X and Pahan K: Up-regulation of BDNF in astrocytes by TNF-alpha: a case for the neuroprotective role of cytokine. J Neuroimmune Pharmacol 1: 212-222, 2006.

11. Nawashiro H, Martin D and Hallenbeck JM: Inhibition of tumor necrosis factor and amelioration of brain infarction in mice. J Cereb Blood Flow Metab 17: 229-232, 1997.

12. Hallenbeck JM: The many faces of tumor necrosis factor in stroke. Nat Med 8: 1363-1368, 2002.

13. Pan W, Ding Y, Yu Y, et al: Stroke upregulates TNFalpha transport across the blood-brain barrier. Exp Neurol 198: 222-233, 2006.

14. Zhu P, Genc A, Zhang X, et al: Heterogeneous expression and regulation of hippocampal prostaglandin E2 receptors. J Neurosci Res 81: 817-826, 2005.

15. Saha RN, Ghosh A, Palencia CA, et al: TNF-alpha preconditioning protects neurons via neuron-specific up-regulation of CREB-binding protein. J Immunol 183: 2068-2078, 2009.

16. Roqué PJ, Guizzetti M, Giordano G and Costa LG: Quantification of synaptic structure formation in cocultures of astrocytes and hippocampal neurons. Methods Mol Biol 758: 361-390, 2011.

17. Wang R, Zhang X, Zhang J, et al: Oxygen-glucose deprivation induced glial scar-like change in astrocytes. PLoS One 7: e37574, 2012.

18. Schmidt OI, Morganti-Kossmann MC, Heyde CE, et al: Tumor necrosis factor-mediated inhibition of interleukin-18 in the brain: a clinical and experimental study in head-injured patients and in a murine model of closed head injury. J Neuroinflammation 1: 13, 2004.

19. Wang XY, Chen ZH, Zhang RY, et al: Construction of a eukaryotic expression vector $\mathrm{pEGFP-C1-BMP-2}$ and its effect on cell migration. J Zhejiang Univ Sci B 13: 356-363, 2012.

20. Durukan A and Tatlisumak T: Preconditioning-induced ischemic tolerance: a window into endogenous gearing for cerebroprotection. Exp Transl Stroke Med 2: 2, 2010.

21. Dirnagl U, Becker K and Meisel A: Preconditioning and tolerance against cerebral ischaemia: from experimental strategies to clinical use. Lancet Neurol 8: 398-412, 2009.

22. Schaller B: Ischemic preconditioning as induction of ischemic tolerance after transient ischemic attacks in human brain: its clinical relevance. Neurosci Lett 377: 206-211, 2005.

23. Castillo J, Moro MA, Blanco M, et al: The release of tumor necrosis factor-alpha is associated with ischemic tolerance in human stroke. Ann Neurol 54: 811-819, 2003.

24. Wang X, Li X, Erhardt JA, et al: Detection of tumor necrosis factor-alpha mRNA induction in ischemic brain tolerance by means of real-time polymerase chain reaction. J Cereb Blood Flow Metab 20: 15-20, 2000.

25. Glazner GW and Mattson MP: Differential effects of BDNF, ADNF9, and TNFalpha on levels of NMDA receptor subunits, calcium homeostasis, and neuronal vulnerability to excitotoxicity. Exp Neurol 161: 442-452, 2000.

26. Watters $\mathrm{O}$ and $\mathrm{O}^{\prime}$ Connor JJ: A role for tumor necrosis factor- $\alpha$ in ischemia and ischemic preconditioning. J Neuroinflammation 8: 87, 2011.

27. Watters O, Pickering M and O'Connor JJ: Preconditioning effects of tumor necrosis factor- $\alpha$ and glutamate on calcium dynamics in rat organotypic hippocampal cultures. J Neuroimmunol 234: 27-39, 2011.

28. Davis DP and Patel PM: Ischemic preconditioning in the brain. Curr Opin Anaesthesiol 16: 447-452, 2003.

29. Weller ML, Stone IM, Goss A, et al: Selective overexpression of excitatory amino acid transporter 2 (EAAT2) in astrocytes enhances neuroprotection from moderate but not severe hypoxia-ischemia. Neuroscience 155: 1204-1211, 2008.

30. Tilleux S, Goursaud S and Hermans E: Selective up-regulation of GLT-1 in cultured astrocytes exposed to soluble mediators released by activated microglia. Neurochem Int 55: 35-40, 2009.

31. Su ZZ, Leszczyniecka M, Kang DC, et al: Insights into glutamate transport regulation in human astrocytes: cloning of the promoter for excitatory amino acid transporter 2 (EAAT2). Proc Natl Acad Sci USA 100: 1955-1960, 2003. 